\title{
Mano zamba radial con radio presente, reporte de un caso, propuesta de manejo quirúrgico y revisión de la literatura
}

\section{Radial Club Hand with the Whole of Radius Present, a Case Report, Proposal of Surgical Management and Literature Review}

\author{
Jairo Fernando Gómez Ramírez ${ }^{1}$ Francisco Javier Lores García ${ }^{2}$ José Tomás Martínez Villegas² \\ ${ }^{1}$ Ortopedia y Traumatología, Cirujano de mano, Colombia \\ 2 Residente de Ortopedia y Traumatología, Universidad Nacional de \\ Colombia, Colombia \\ Address for correspondence José Tomás Martínez Villegas, Residente \\ de Ortopedia y Traumatología, Universidad Nacional de Colombia, \\ Colombia (e-mail: jtmartinezv@unal.edu.co).
}

Rev Iberam Cir Mano 2018;46:131-134

\section{Resumen}

Palabras clave

- mano zamba radial

- osteotomía de radio

- deformidades congénitas del miembro superior
Introducción Se describe una forma inusual de deformidad congénita de la mano, que consiste en una deformidad en mano zamba radial con radio presente pero luxado en extremos proximal y distal. Es una variedad infrecuente, por lo que proponemos una opción de corrección quirúrgica. Solo existe un caso descrito en la literatura con la característica de tener radio presente completo y no existen reportes de tratamiento, por tanto, ese sería el primer reporte de un tratamiento.

Materiales y Métodos Reporte de caso de un paciente de 4 años de edad con mano zamba radial con radio presente congénita, valorado en un hospital de nivel III en la ciudad de Bogotá, Colombia, el cual fue llevado a corrección quirúrgica en el año 2010. Se realizó una osteotomía de acortamiento del radio con cierre del espacio radiocubital distal, capsulotomía ulno carpiana con reinserción de la cápsula al radio distal y centralización del carpo sobre el remanente del radio.

Resultados Paciente con corrección de la deformidad radiológica y clínicamente con mejoría del agarre en seguimiento realizado en el primer mes de posoperatorio.

Conclusiones Se puede realizar una corrección quirúrgica de la deformidad en mano zamba radial con radio presente, utilizando la técnica quirúrgica descrita.

Introduction An unusual form of congenital deformity of the hand is described, which consists of radial club hand deformity with the whole of the radius present but dislocated at proximal and distal ends. It is an infrequent variety, so we propose a surgical correction option. There is only one case described in the literature with the characteristic of having complete radio present and there are no reports of treatment, therefore, that would be the first report of a treatment. received

September 4, 2017

accepted

March 1, 2018

published online

May 14, 2018
DOI https://doi.org/

10.1055/s-0038-1641158. ISSN 1698-8396.
Copyright $\odot 2018$ Thieme Revinter

Publicações Ltda, Rio de Janeiro, Brazil
License terms

(c) (i) $\ominus$ (\$) 
Material and Methods The 4-year-old patient was evaluated in our hospital and taken to surgical correction, a shortening osteotomy of the radius was performed with closure of the distal radioulnar space, ulnar carpal capsulotomy with reinsertion of the capsule to the distal radius and centralization of the carpus over the radius.

Results In the first month of the postoperative period, the correction of the deformity is maintained, checking it radiologically and clinically there is improvement of the grip. Conclusions Surgical correction of the deformity in the radial club hand with radius, using the described surgical technique.

\section{Introducción}

Se describe un caso de deformidad congénita de la mano, la cual consiste en deformidad en mano zamba radial con todo el radio presente pero luxado en los extremos proximal y distal. En la literatura solo existe un caso descrito por Waldram ${ }^{1}$ pero no aporta tratamiento, por lo cual ése sería el segundo caso reportado y el primer reporte de un tratamiento para esa variedad de la deformidad, la cual no es posible ubicar dentro de la clasificación de anormalidades congénitas de la mano. ${ }^{2}$

\section{Caso clínico}

Reporte de caso de un paciente primogénito, género masculino, sin antecedentes patológicos ni perinatales relevantes, padres sin antecedentes de deformidades congénitas de las manos. Valorado e intervenido a los 4 años de edad, la evaluación clínica y radiográfica evidencia una forma infrecuente de mano zamba radial, con la característica particular de tener todo el radio presente pero luxado. La deformidad de ese paciente consiste en una desviación radial del carpo, con luxación distal del radio, cinco dedos funcionales y un apéndice radial que parecía un pulgar accesorio, pero en realidad se trataba del radio luxado. (-Fig. 1). Las imágenes radiológicas confirman la deformidad descrita ( - Fig. 2). En el prequirúrgico el rango de flexoextensión de los dedos y la muñeca era completamente normal, pero con desviación radial de la mano y disminución de la fuerza, con limitación completa de la pronosupinación. No fue posible establecer rangos de movilidad intraoperatoria considerando la inmovilización con el clavo descrito.

\section{Técnica quirúrgica}

Vía de abordaje palmar, disección del nervio mediano, se aprecia nervio mediano, tendón del braquiradialis y flexor carpi radialis presentes insertados en el radio luxado, flexores de los dedos en posición habitual y sin compromiso, no se realiza intervención sobre los extensores. Se identifica extremo distal del radio libre en el apéndice de piel descrito y se identifica la arteria interósea anterior y los ramos nutrientes que irrigaban el segmento distal del radio. Se realiza tenotomía del braquiradialis a nivel de la inserción en

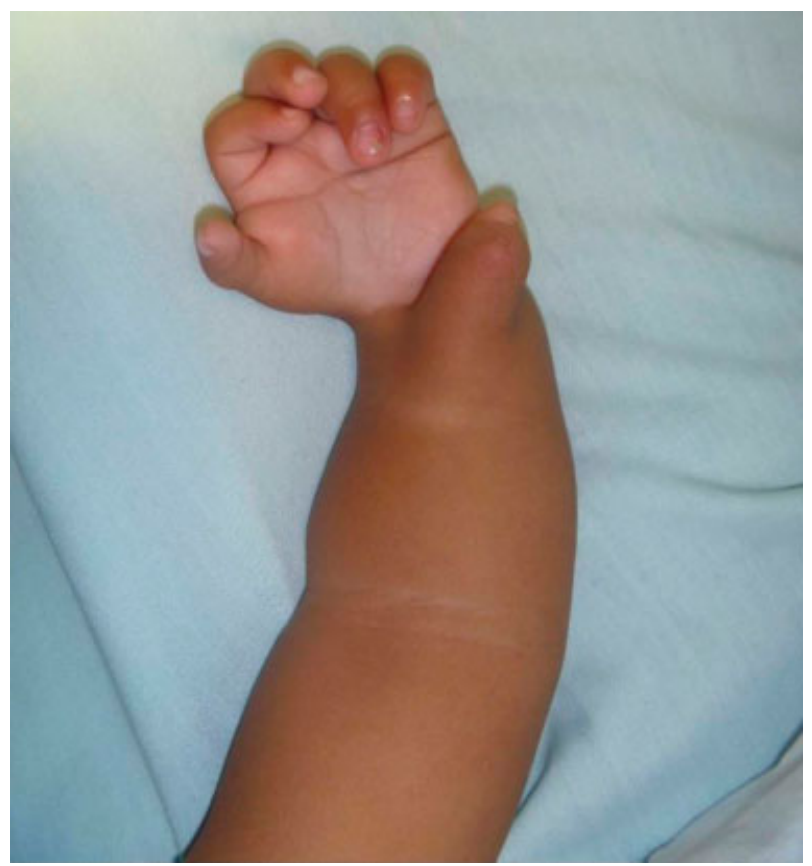

Fig. 1 Imagen Clínica de deformidad en mano zamba radial.

el radio, para poder manipular el extremo distal del radio. Se levantó además un colgajo del periostio con sus vasos manteniendo la irrigación del segmento distal del radio, debido a que la longitud mayor del radio no permitía centralizarlo, se realizó una osteotomía de acortamiento del mismo a nivel diafisiario, realizando una resección de un segmento de $2 \mathrm{~cm}$ del radio para igualar su altura con la del cúbito (-Fig. 3), tras realizar la osteotomía se realiza capsulotomía ulnocarpiana y se pudo centralizar el radio con el carpo y fijar el radio y la muñeca con la misma aguja de kirschner ( - Fig. 4C), se sutura la cápsula al extremo distal del radio, el braquiradialis se fijó con sutura a la capsula articular. Se remodela además la piel redundante del apéndice descrito con la cual se logra cobertura del defecto cutáneo ( - Fig. 4A-B). Se deja inmovilización en el posquirúrgico con férula antebraquiopalmar en posición funcional, inmovilizado durante 8 semanas, no se indicó rehabilitación considerando la edad del paciente (pediátrico), retirada del clavo a las 6 semanas posterior a las cuales el paciente nunca retorna a la 


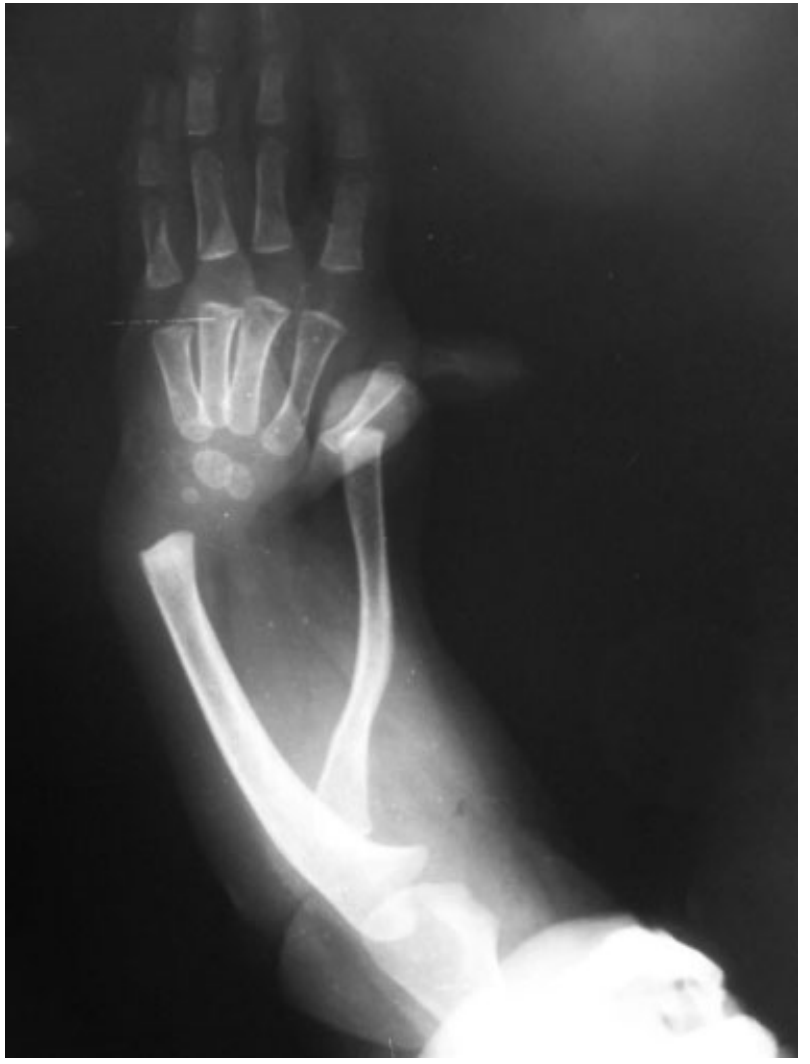

Fig. 2 Radiografía de Deformidad infrecuente, mano zamba radial con radio presente.

consulta, por ser de zona selvática no es posible localizarlo para continuar el seguimiento.

\section{Discusión}

Se realizó una revisión de la literatura con el fin de buscar otros casos reportados de este tipo particular de mano zamba

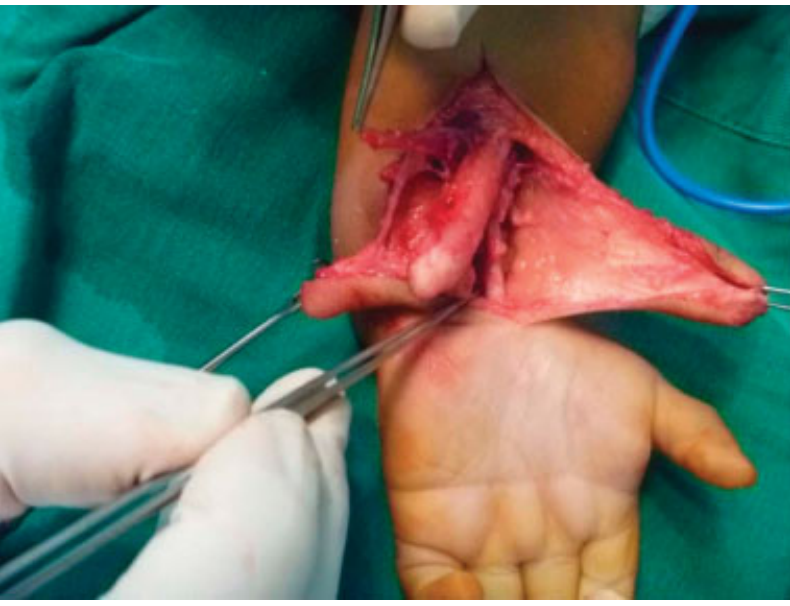

Fig. 3 Imagen intraoperatoria de radio luxado.

radial, y posibilidades de tratamiento. Esa es una presentación inusual de la mano zamba radial, en la cual encontramos el radio presente y completo pero luxado, y no es posible clasificarla dentro de la clasificación de las malformaciones congénitas de la mano y la extremidad superior. Solamente existe un caso reportado en la literatura y no existen estudios que describan algún tipo de tratamiento para esa presentación de la deformidad, por lo que no es posible realizar una comparación con otras técnicas. La centralización del cúbito es el procedimiento quirúrgico estándar para el manejo de la mano zamba radial, sin embargo, en ese caso, el radio estaba presente, razón por la cual se decidió reposicionarlo, pero debido a que era más largo se optó por realizar una osteotomía de acortamiento del radio y al tenerlo libre distalmente conseguimos preservar la circulación de ese segmento identificando la arteria interósea o intraósea y conservando la circulación perióstica.
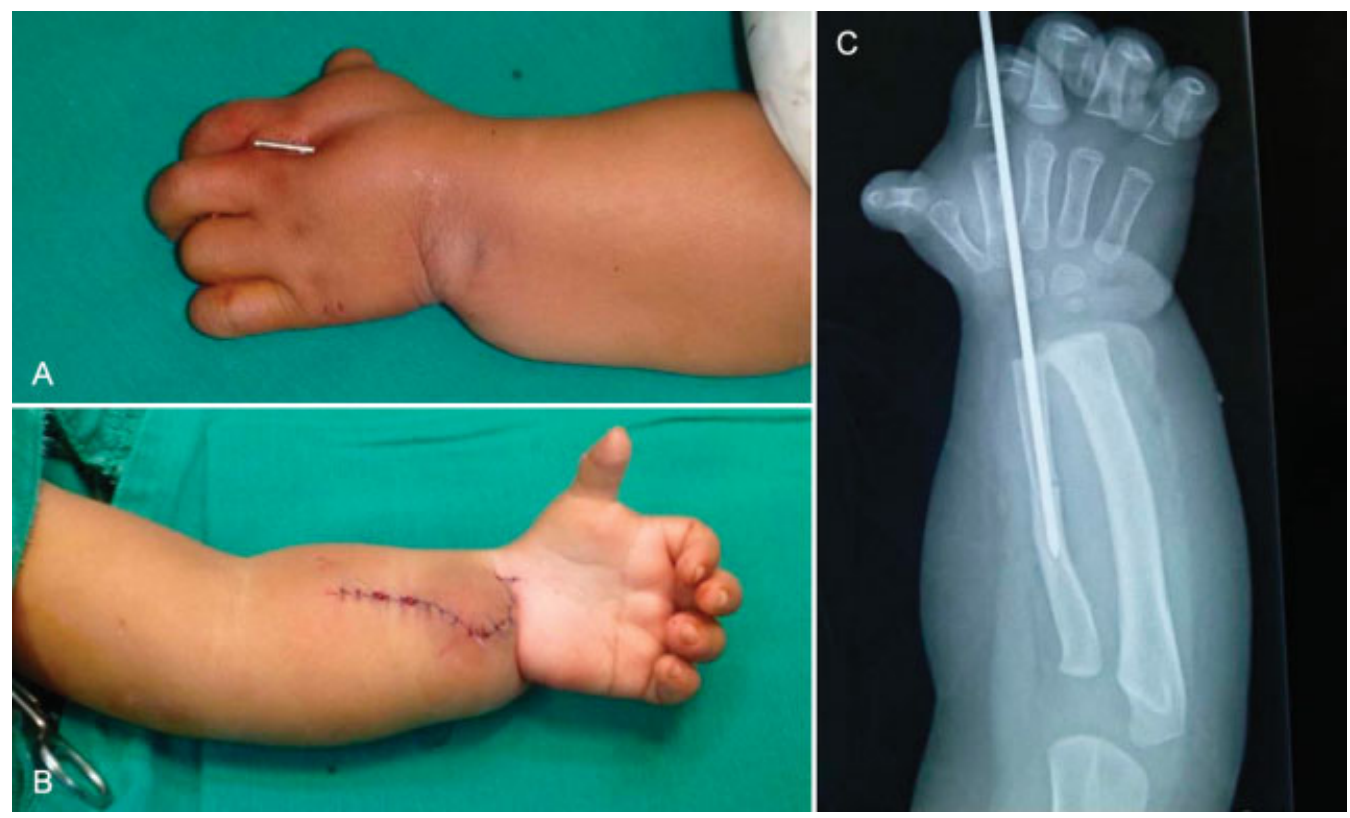

Fig. 4 (A-B) Resultado clínico posoperatorio. (C) Resultado radiológico posoperatorio. 
134 Mano zamba radial con radio presente Gómez y col

\section{Conclusiones}

La mano zamba radial con radio presente luxado, se puede corregir mediante osteotomía de acortamiento del radio, con lo que se logra una adecuada centralización del mismo sobre el carpo.

\section{Bibliografía}

1 Waldram MA. An unusual case of radial club hand. J Hand Surg [Br] 1990;15(02):190-192

2 Tonkin MA, Tolerton SK, Quick TJ, et al. Classification of congenital anomalies of the hand and upper limb: development and assessment of a new system. J Hand Surg Am 2013;38(09):1845-1853 